\title{
A REVIEW PAPER ON MICROSOFT HOLOLENS
}

\author{
Shaikh Adiba Kashish \\ Department of CSE \\ PES College of Engineering, Aurangabad, \\ Maharashtra, India
}

Abstract-We are able to connect with threedimensional holograms with our real world. But now we have a totally new medium to express our creativity and learn new ways of visualization. There you don't need to touch the screen or click anything on the mouse and use only gestures to create any type of shape and size of holograms. With the help of eyes, we can navigate. Now we can use our voice to communicate with applications. Microsoft HoloLens understand our voice movement and vision, and it is enabled to interact with us. The hololens completely take it to the next level where our digital content will be as real as our physical objects with the help of holograms. The Microsoft HoloLens have high definition lenses and spatial sound with the help of that we are able to hear hologram in real world. with the advanced sensors and HPU (holographic processing unit) helps to understand the world around you. If we have a new idea we can show it with many perspectives in microsoft holoLens we can pin our holograms in the physical environment and also can provide a new way to observe the world.

Keywords - Holograms, Microsoft HoloLens, Virtual Reality (VR), Augmented Reality (AR), Holographic Processing Unit(HPU), Three Dimensional(3D), Virtualization, Mixed Reality (MR)

\section{INTRODUCTION}

Microsoft HoloLens is the first fully untethered Holographic computer and It's enabling us to place, view, and interact with holograms in our physical environment. it has more sensors. A custom holographic processing unit, batteries, integrated cooling, speakers, and high definition stereoscopic3D optical head-mounted display. Microsoft HoloLens is also known as project Baraboo (in-development). it is developed and manufactured by Microsoft. it will allow viewing the world the 2D or 3D content without sitting in front of the computer. It's trying to fetch the best features from both Augmented reality (AR) and Virtual reality (VR). VR is the 3Dimensional computer-generated environment and provided the effects of concrete existence without actually having it and with those immersions of vision, it also provides sound and tactile feedback. That means a person totally can escape from the real world by embracing it in the cyber world. This is the new form of human-computer interaction which is beyond the mouse, keyboard, or even touch screens and implemented on gloves,

\author{
Nidhi Ghodele \\ Department of CSE \\ PES College of Engineering, Aurangabad, \\ Maharashtra, India
}

headsets, or helmets such types of interactive devices. On the other AR just supplements reality and not completely replace it. AR allows the user to see the real world with its virtual object superimposition composited with the real world. Microsoft HoloLens goes beyond augmented reality and virtual reality by providing people the three Dimensional holograms blended with the real world when you share your ideas you can show and analyze from the multiple perspectives.

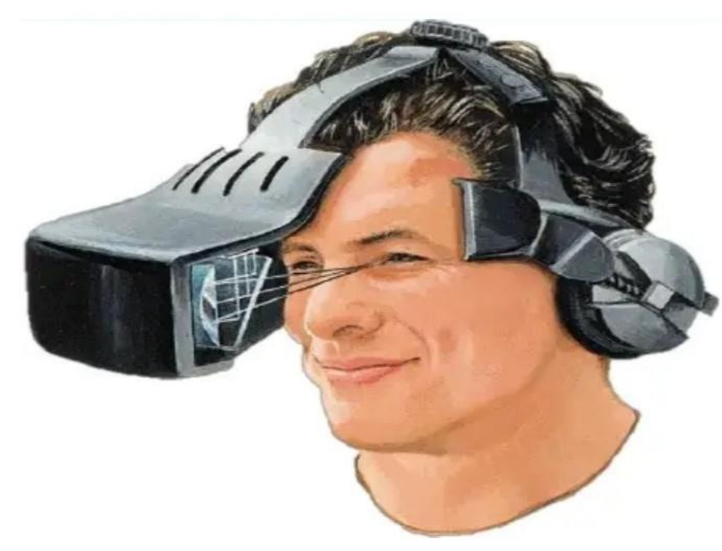

fig 1 : A gadget use for Virtual Reality

\section{DESCRIPTION}

The hologram is the next evolution in the computing world. With the holography technique, hardware, software creators develop a new holographic platform. Microsoft Hololens with Window 10it is the first head-mounted display run windows mixed reality (WMR) platform and the product family is under windows 10 operating system. its type is Mixed reality augmented reality head-mounted display smartglasses. The release date is March 30, 2016 (Development Editon), and (Development Edition 2) announced May 2, 2019. The introductory price starts at $\$ 3000$. CPU used is intel 32-bit (1 $\mathrm{GHz}$ ) and the memory is 2 GB RAM and 1 GB HPU RAM. the storage is 65 GB (flash memory) with a 2.3-megapixel widescreen display and Spatial sound technology. The inertial measurement unit used in this is Accelerometer, gyroscope, and magnetometer with 4 sensors. The Controller input is Gestural commands via sensors and HPU (Holographic processing units). it is totally untethered that means no wires, no phone, or even connection to PC is needed. [1] Microsoft hololens allows 


\section{International Journal of Engineering Applied Sciences and Technology, 2020 \\ Vol. 5, Issue 7, ISSN No. 2455-2143, Pages 232-236 \\ Published Online November 2020 in IJEAST (http://www.ijeast.com)}

you to pin holograms in your physical environment and provides a new way to see our world. With these holographic APIs, the AR features can readily implement on wide range of windows 10 devices.

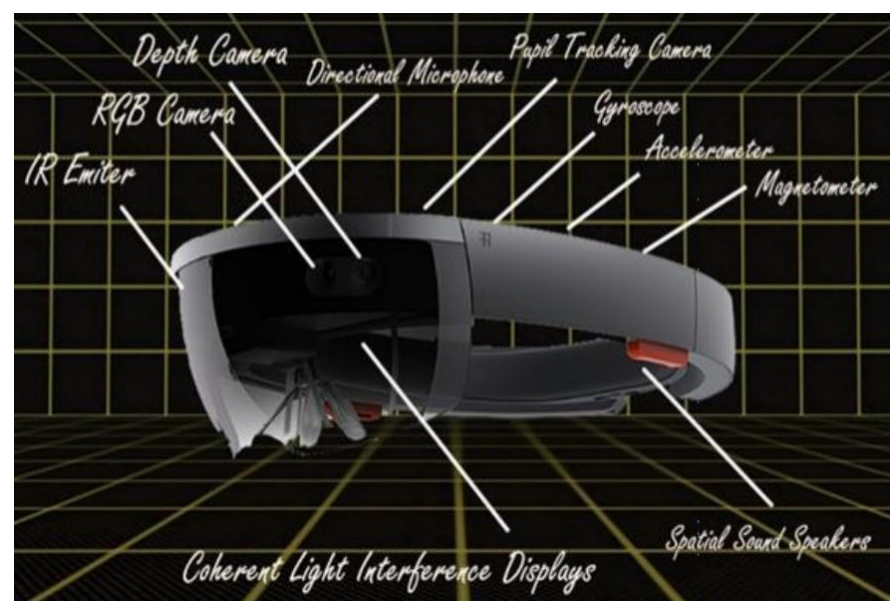

Fig 2.: Parts of Microsoft HoloLence

\section{SPECIFICATIONS}

OS - Windows 10

CUP - Intel Atom x5-Z8100, 1.04 GHz. Intel Airmont (14nm), 4 Logical Processor

GPU/HPU - HoloLens Graphic

GPU Vendor ID - 8086h (Intel)

Dedicated Video Memory - 114MB

Shared System Memory - 980 MB

RAM - 2GB

Storage - 64GB (54.09 GB available)

App Memory Usage Limit - 900 MB

Battery - $16.500 \mathrm{mWh}$

Camera Photos - 2.4 MP (2048x1152)

Camera Video - 1.1 MP (1408x792)

Video Speed - 30 FPS

The microsoft hololens use new advanced working sensors and a new Holographic Processing Unit (HPU) that can help to understand the world around the user.[2] holograms are totally made of light and that holographic model can be viewed from distance and from different angles like a physical object and they do not offer any physical resistance when they touched or pushed because they are massless.

The holograms are two dimensional like a screen of TV and it also can be three dimensional like the real object in the world. In the microsoft hololens the holograms can appear like the real world and we are able to move it, shape it or change it according to the physical environment. It has 4 or more cameras just to track the environment of the user and also to project the holograms on the surface. And high definition sensors are used to identify the movement of the user.

\section{WORKING}

The user interface of Microsoft holoLens takes voice, graze and gestures as an input command and internal computer works on these commands. The holoLens is equipped with a holographic processing unit (HPU). This processing unit is a specialized electronic circuit designed and it is as a co-processor clip (along with GPU and CPU ) used to process. The mean task of HPU's try to detect the place around it.

According to Microsoft the HPU is capable of, "processing terabytes (TB) of information from its different sensors in the real world. The holoLens has the three transparent layer of lenses and those lenses help to view the holographic object in reality. This device just auto-adjust to user's eye pitch and built the image of the object accordingly. The camera of the holoLens looks at the room so it can identify the physical object present in the room and then uses that information to project the image on the top of that physical object or even inside them.

The HPU processes the information from these cameras to identify that any physical object present in the room and at what distance it is present and also the shape, size, and position the object. [4] The holoLens have a number of sensors the sense our movement in the room and this data with colored glass to create images that can easily interact with us according to our movement in a different direction.

The input command from the users is taken by the sensors for a better understanding of the surrounding. It uses a different types of motion trackers like gyroscope, accelerometer, and magnetometer specially to check user's head motions. Some sensors are use identify the intensities of the light, Motion sensor to read the actions of the user and the IR sensors are use to detect any obstacle present near the user. GPS for the location. The Microsoft holoLens can even able to capture the slight movement of user and acts accordingly. 


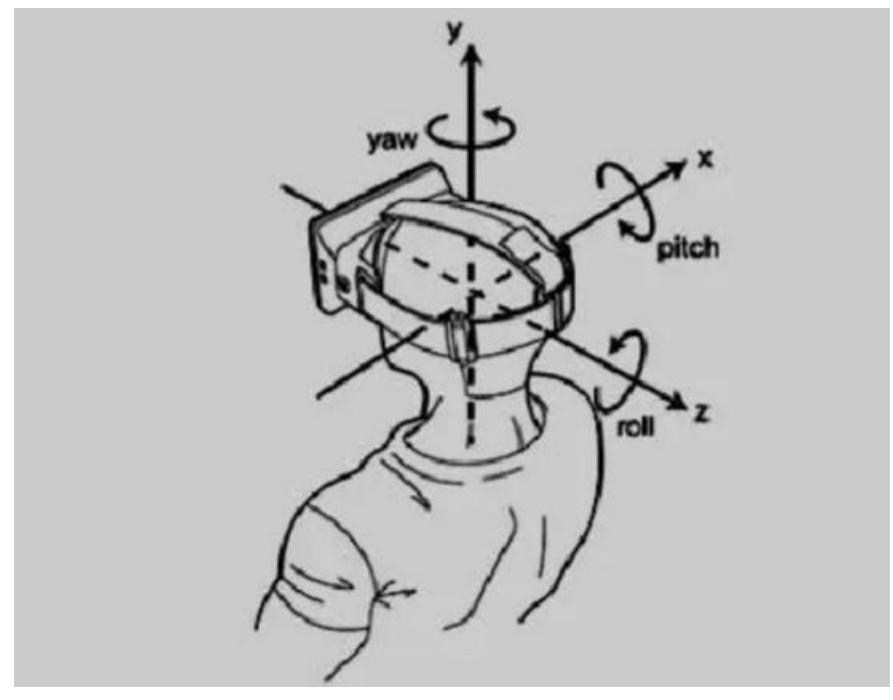

Fig 3: Sensing head Movement

\section{COMPARison Between Microsoft Hololens1 And HOLOLENS2}

The following is the list of comparisons on the different specs of Microsoft Hololens1 and Microsoft Hololens2.

1)The Price on release day for Hololens1 is $\$ 3000$ for the developers and $\$ 5000$ for commercial suite and for Hololens2 is $\$ 3500$ or $\$ 125 / \mathrm{mo}$ for enterprise or $\$ 99 / \mathrm{mo}$ for the developer. 2)The Display Resolution per eye for Hololens 1 is $1280 \times 720$ and Hololens2 is

\section{$2048 \times 1080$.}

3)The Holographic density in radians for Hololens1 is less than $2.5 \mathrm{k}$ (light points per radians) and Hololens 2 is also the same.

4)The FOV (field of view) in degrees for Hololens1 is 34degrees and Hololens 2 is 52 degrees.

5)The Weight in grams for Hololens1 is $579 \mathrm{gm}$ and Hololens 2 is $566 \mathrm{gm}$.

6)The IPD Adjustment is available for the holograms.

7)The Eye based rendering for [5] Hololens1 is of the type Automatic pupillary distance calibration and Hololens 2 is of the type Display optimization for 3D eye position.

8)The eye tracking is not available for Hololens 1 while it is available for Hololens2.

9)The processor used for Hololens1 is Intel 32-bit(1GHz) and for Hololens2 Qualcomm Snapdragon 850.

10)The camera used for Hololens1 is 2.4MP having HD video while for Hololens 2 is *MP having 1080 p video.

11)The Audio for Hololens1 has built-in speakers having a $3.5 \mathrm{~mm}$ jack while Hololens 2 has built-in spatial sound also has $3.5 \mathrm{~mm}$ jack.

12)Microsoft Hololens 1 has a four-microphone array while the second version is having a five microphone array.
13)The Hololens1 doesn't have a flip-up visor while the second version has it.

14)Voice command is available for both the versions.

15)Biometric security i.e Iris scanning isn't available for Hololens 1 while it is for Hololens2.

16)One hand tracking is available for the first version while Both hands full tracking is there for the second version.

17)Gestures such as Bloom, Air tap, tap and hold is available for both the versions.

18)Also, Gestures such as press, Grab, Direct Manipulation, Touch interaction, and scroll with the wave isn't available for Hololens1, on the other hand, it is available for Hololens2

\section{FUTURE SCOPE}

Microsoft revealed HoloLens, an augmented reality headset aimed at blending the physical and digital worlds in the year 2015. The headset would have its own graphics processing and it allows the user to move freely instead of being tethered, and the demo video showed various uses from watching Netflix as if it is projected on a wall, to be working on 3D product designs. Microsoft's goal with the HoloLens is to not merely create another piece of hardware kit; but rather, give rise to everything that we do on two-dimensional screens into the real world with an immersive blended reality experience.

Unlike other virtual reality headsets, HoloLens allows you to interact and manipulate holograms by introducing these holograms into your physical environment without having any change in the real world. With Microsoft's HoloLens, the possibilities are considerably more positive. By integrating virtual objects into our actual physical world, HoloLens presents us with the opportunity to improve our reality, rather than replace it.it is done with the help of integration of the virtual objects into our actual physical world.

The old version HoloLens already had a wide range of use case, but due to some technical limitations like the heaviness and view, the full productivity was never reached. All these drawbacks have been solved by Microsoft Hololens2, so the potential benefits are broader and enhanced than the first version.

HoloLens is useful in army battlefield, where detailed instruction can be given to the untrained army officers in the midst of any combat it is also used in education with the help of 3D projectors in engineering for teaching and learning a different kind of engineering training entertainment like mixed reality which is not only reserved for gaming and filming. it is most useful in health care for viewing different surgeries and also for the anatomical studies with 3D holograms and finally in infrastructure to preview the models of the big construction. 
International Journal of Engineering Applied Sciences and Technology, 2020

Vol. 5, Issue 7, ISSN No. 2455-2143, Pages 232-236

Published Online November 2020 in IJEAST (http://www.ijeast.com)

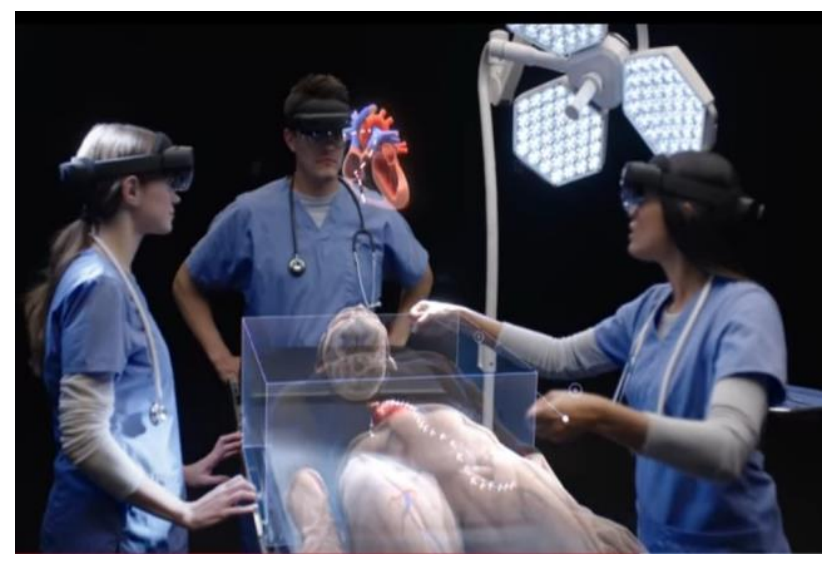

Fig. 4 HoloLens user can see representation of a human body in 3D, and can navigates through the layers of skin, muscle, blood vessels, and organs to the skeleton below.

\section{CONCLUSION}

The holographic experience with Microsoft hololens are the different Microsoft holoLens as both augmented and virtual reality. And it's going to prove very useful in the future. Microsoft has never before launched any product in hololens technology to make a strict conclusion on this technology is very hard. There is so much potential that the HoloLens can offer. Their more applications on Microsoft hololens that will explore in the future then the production cost will reduce as the technology improves and it will become prevalent in our lives.

The hololens came as an idea and evolved so much into a creation that will soon change the different perceptions in life. those technologies are real-life fun but people gradually stop leaving their life and also taking care of small sensors is very hard. always need complicated positioning. people can also explore new places it is a totally new way of learning and teaching. At some time it is very useful like using during medical emergencies and at the workplace, the employees can work together from anywhere like the new vision of work. The performance of hololens is far ahead then all existing technologies and with such an outward potential of hololens we can't predict where the limit of holoLens will stop in the future.

\section{REFERENCE}

[1](Sri Krishna College of Arts and Science) Ms. S. Karthika, P. Praveena, M.GokilaMani HOLOLENC IJCSMC, Vol. 6, Issue. 2, February 2017, pg.41-50 https://docs.google.com/document/d/1tif4wxpOFQJUZLTUT HIEHf6XOTa GmFIvoeow8rHSxeE/edit

[2]( (PDF) Microsoft HoloLens Seminar Report | Navaneeth Krishnan Karimbil - Academia.edu
https://www.academia.edu/28726871/Microsoft_HoloLens_Se $\underline{\text { minar_Report }}$

\section{[3]HITESH KUMAR SINGH( SEMINAR REPORT ON MICROSOFT}

HOLOLENS)https://www.slideshare.net/hiteshkumarsingh10/ microsoft-holol ens-seminar-report

[4]Mixed reality in production and logistics: Discussing the application potentials of Microsoft HoloLensTM Sebastian Langa,*, Mohammed Saif Sheikh Dastagir Kotaa, David Weigerta,b, Fabia in production and logistics: Discussing the applicatio

https://www.sciencedirect.com/science/article/pii/S187705091 930122X

[5]HoloLens 2 vs HoloLens 1 - Should I upgrade my device? https://4experience.co/hololens-2-vs-hololens-1-whatsnew/\#: :text=The $\% 2$

Obasics,2GB\%20in\%20HoloLens\%201).

[6]Microsoft HoloLens 2: Partner Spotlight with Philips https://m.youtube.com/watch?v=loGxO3L7rFE

[7]https://en.wikipedia.org/wiki/Microsoft_HoloLens

[8]Simon N. Leonarda* and Robert N. Fitzgeraldb ORIGINAL RESEARCH

ARTICLE Holographic learning: A mixed reality trial of Microsoft HoloLens in an Australian secondary school (Received: 24 September 2018; final version received: 28 October 2018)

file://C:/Users/HP/Downloads/2160-Article\%20Text-113071-10-20181127\% 20(4).pdf

[9] https://www.researchgate.net/publication/321587492_T echnical_Evalu ation_of_HoloLens for_Multimedia_A_First_Look

[10]https://www.researchgate.net/publication/322849028 Augmented_Re ality_Technology_Using_Microsoft_HoloLens_in_Anatom ic_Pathology

[11]https://lib.dr.iastate.edu/cgi/viewcontent.cgi?article $=73$ $\underline{85 \& \text { context }=\text { et }} \underline{\mathrm{d}}$

[12]https://www.academia.edu/Documents/in/HoloLens-1

[13] https://ieeexplore.ieee.org/document/7836494

[14] $\underline{\text { https://journal.alt.ac.uk/index.php/rlt/article/download }}$ /2160/pdf_1/ 
International Journal of Engineering Applied Sciences and Technology, 2020

Vol. 5, Issue 7, ISSN No. 2455-2143, Pages 232-236

Published Online November 2020 in IJEAST (http://www.ijeast.com)

[15]

$\underline{85 \& \text { context}=e t} \underline{\text { d }}$

[16]https://designawards.core77.com/Consumer-

Product/51416/Microsof t-Hololens

[17]https://venturebeat.com/2017/02/13/microsofts-

spectator-view-shows -what-hololens-users-see-from-

third-person-perspective/

[18]https://www.microsoft.com/en-us/hololens

[19]https://docs.google.com/document/d/1fejZdtAp7aUThe

S_BAOQuUSb

$\underline{\text { A7Ho4pnoSGkYqaiEe8k/edit?usp=drivesdk }}$

[20]https://www.academia.edu/28726871/Microsoft_HoloL ens_Seminar

$\underline{\text { Report }}$ 